\title{
Eine Klinik stellt sich vor - die Universitäts-Hautklinik Kaunas, Litauen
}

\section{A Clinic Presents itself - The University Clinic for Dermatology Kaunas, Lithuania}

Autor

Institut

\section{S. Valiukeviciene}

Klinik für Dermatologie und Venerologie, Litauische Universität für Gesundheitswesen, Kaunas, Litauen
Bibliografie

DOI http://dx.doi.org/

$10.1055 / \mathrm{s}-0034-1377876$

Akt Dermatol 2014; 40: 393-396

(c) Georg Thieme Verlag KG

Stuttgart · New York

ISSN 0340-2541

Korrespondenzadresse

Prof. Skaidra Valiukeviciene Leiterin der Klinik für

Dermatologie und Venerologie

Litauische Universität

für Gesundheitswesen

Eiveniu 2

Kaunas LT-50009

Litauen

Skaidra.valiukeviciene@

kaunoklinikos.It

\section{Zusammenfassung \\ $\nabla$}

Die erste Klinik für Haut- und Geschlechtskrankheiten wurde an der Vytautas-Magnus-Universität in Kaunas im Jahr 1923 gegründet. Im Jahr 2001 wurde die Universitäts-Hautklinik Kaunas im Klinikum des interdisziplinären Universitätskrankenhauses Kaunas untergebracht. Seit dieser Zeit hat die Zusammenarbeit mit deutschsprachigen Dermatologen und das interdisziplinäre Konzept des Kaunas-Klinikums den Aufbau der Uni-

\section{Einleitung}

$\nabla$

Kaunas ist die zweitgrößte Stadt Litauens mit etwa vierhunderttausend Einwohnern. Die bewegte Geschichte von Kaunas begann am Zusammenfluss von zwei Flüssen, Memel (Nemunas) und Neris, im 13. Jahrhundert. Seither ist Kaunas als eine wichtige Hansestadt bekannt und auch als vorübergehende Hauptstadt (1920-1939). Die Stadt ist bis zum heutigen Tag ein wichtiges Industrie- und Kulturzentrum und als Studentenstadt etabliert. Im Jahre 1923 wurde in der ein Jahr zuvor gegründeten Universität Kaunas die Klinik für Haut- und Geschlechtskrankheiten eröffnet (Ambulanz und 25 Betten) [1]. Kurz vor dem Zweiten Weltkrieg begann die Medizinische Fakultät der Universität Kaunas mit dem Bau eines neuen Klinikgebäudes nach dem Entwurf des berühmten französischen Architekten Urbain Cassan (1890-1979). 1940 wurde die Universitätsklinik für Haut- und Geschlechtskrankheiten eingerichtet (Ambulanz und 49 Betten). Nach dem Zweiten Weltkrieg war die Klinik in städtischen „Dispensaires“ untergebracht (Ambulanz und 120 Betten) [2]. In der Sowjetzeit war die Tätigkeit der Dermatologen primär durch die Kontrolle der Geschlechtskrankheiten bestimmt. Der internationale Austausch war gering, die dermatovenerologische Lehre und Forschung erlebte einen Rückgang.
versitäts-Hautklinik sehr stark beeinflusst. So entwickelten sich neue Fachbereiche wie die Dermatoonkologie, die Dermatochirurgie und die Fotochemotherapie an der Universitäts-Hautklinik Kaunas. Die stetig wachsende Anzahl der dermatologischen Patienten, der Studenten und „Residents“ zeigt, dass die Universitäts-Hautklinik Kaunas ein wichtiges regionales Zentrum der dermatologischen und venerologischen Versorgung wurde.

\section{Schwerpunkte der klinischen Tätigkeit $\nabla$}

Im Jahr 2001 zog die Hautklinik mit den Dozenten zurück in das interdisziplinäre Universitäts-Krankenhaus Kaunas-Klinikum (Ambulanz, 16 vollständige und 4 teilstationäre Betten). Ein Teil der praktisch tätigen Dermatologen wurde auf einer dermato-venerologischen Station des städtischen Krankenhauses untergebracht (20 stationäre und 20 teilstationäre Betten). Seit dieser Zeit haben die Zusammenarbeit mit deutschsprachigen Dermatologen und die interdisziplinären Möglichkeiten auf dem Gelände des Klinikums die Entwicklung der Universitäts-Hautklinik Kaunas sehr stark beeinflusst ( Tab. 1).

In den letzten zehn Jahren nahm die Zahl der Patienten in der Universitäts-Hautklinik Kaunas deutlich zu. In der dermatologischen Ambulanz fanden im Jahr 200210265 und 201324887 Patientenkontakte statt. Die Erstvorstellungen (Konsultationen) betrugen im Jahr 20025582 und 201312431 Patienten. Die meisten Patienten waren aus der Stadt oder dem Gebiet von Kaunas (90\%) und 10\% aus anderen Regionen Litauens. Auf der Station wurden 2002449 und 2013612 Patienten behandelt. Die durchschnittliche Fallschwere lag in den Jahren 2012 - 2013 bei einem CMI von 1,571 (minimaler und maximaler Wert bei 0,476 und 3,743). Die Bettenauslastung lag bei 65\% im Jahr 2002 und bei 99\% im Jahr 2013. 


\begin{tabular}{|c|c|c|c|}
\hline Dermatologische Bereiche & Gastgebende Institution & Zahl der Hospitanten & Jahr \\
\hline $\begin{array}{l}\text { Dermatoonkologie und } \\
\text { Dermatochirurgie }\end{array}$ & $\begin{array}{l}\text { Otto-von-Guericke-Universität } \\
\text { Magdeburg }\end{array}$ & 2 & 2002,2008 \\
\hline Dermatoallergologie & & 1 & 2004,2014 \\
\hline Dermatohistopathologie & & 2 (1 Pathologe) & 2004,2005 \\
\hline Notfalldermatologie & & 1 & 2007 \\
\hline $\begin{array}{l}\text { Dermatochirurgie und Wächter- } \\
\text { Lymphknoten-Biopsie }\end{array}$ & & 1 (Plastischer Chirurg) & 2005 \\
\hline Hautmykologie & Universität Köln & 1 (Labormediziner) & 2004 \\
\hline $\begin{array}{l}\text { Immunofluoreszenz-Diagnostik } \\
\text { von Dermatosen }\end{array}$ & & 1 (Pathologe) & 2014 \\
\hline
\end{tabular}

Die Entwicklung der neuen dermatologischen Bereiche an der Universitäts-Hautklinik Kaunas im Rahmen der langfristigen Hospitationen der Dermatologen und anderer Spezialisten in deutschsprachigen Ländern.

Die Finanzierung erfolgte durch die Stipendien der Berliner Stiftung für Dermatologie (1 Person), des Deutschen Akademischen Austausch-Dienstes (2 Personen), der Erasmus-Fortbildung und der privaten Stiftung von Prof. Gustav Marhle.

\begin{tabular}{|llll|}
\hline ICD-10-Kodierung & Diagnose & Ambulante & $\begin{array}{c}\text { Stationäre } \\
\text { Kehandlung }\end{array}$ \\
\hline L40 & Psoriasis & 2497 & 228 \\
\hline L23 & Kontaktekzem & 2187 & 110 \\
\hline L30 & Sonstige Dermatitis & 960 & 84 \\
\hline L20 & Atopisches Ekzem & 721 & 33 \\
\hline D17-D23, D28-29, L82 & Gutartige Hauttumoren & 5962 & 0 \\
\hline L57, D03, D04 & Präkanzerosen & 1652 & 0 \\
\hline C44 & Sonstige bösartige & 1458 & 4 \\
\hline C43 & Neubildungen der Haut & & 0 \\
\hline C84, C85 & Bösartiges Melanom & 279 & 9 \\
\hline B35-B49 & Hautlymphome & 177 & 5 \\
\hline B00-B09 & Hautmykosen & 1061 & 8 \\
\hline L70-L71 & Virale Dermatosen & 603 & 0 \\
\hline A52.1; A52.8 & Akne und Rosazea & 1218 & 6 \\
\hline A55-A56 & Neurosyphilis & 33 & 0 \\
\hline A60, A63 & Chlamydien-GK & 53 & 0 \\
\hline A51-53 & Virale GK & 22 & 0 \\
\hline
\end{tabular}

Tab. 2 Die häufigsten Diagnosen der Universitäts-Hautklinik Kaunas (im Jahr 2013).

GK = Geschlechtskrankheiten

Die durchschnittliche Liegezeit betrug 9,38 Tage. In der Tagesklinik ergaben sich im Jahr 20092870 und im Jahr 2013 bereits 3612 teilstationäre Behandlungen. Die Verteilung der häufigsten Diagnosen der Universitäts-Hautklinik sind in $\bullet$ Tab. 2 aufgelistet. Chronische entzündliche Dermatosen wie Psoriasis, Kontaktund atopisches Ekzem sind führende Diagnosen auf der Station. Doch in der Ambulanz verteilen sich die häufigsten Diagnosen auf Hauttumoren, Psoriasis und Kontaktekzem. Die Zusammenfassung aller durchgeführten Prozeduren ist in 0 Tab. 3 dargestellt. Am häufigsten führten die Dermatologen der UniversitätsHautklinik lokale Exzisionen, Biopsien und Lichttherapie durch. Das dermatologische Curriculum in Litauen ist nur auf kleinflächige Dermatochirurgie und diagnostische Dermatoonkologie begrenzt. Die ausgedehnten Exzisionen, Sentinel-LymphknotenBiopsien und die radikalen Lymphadenektomien erledigen im Klinikum Kaunas die plastischen Chirurgen. Doch die allgemeine Versorgung von Patienten mit malignen Hauttumoren erfolgt in enger Zusammenarbeit zwischen Dermatologen, Pathologen und Onkologen.

Ein Schwerpunkt in der Behandlung von Patienten mit chronisch entzündlichen Hauterkrankungen stellt die Systemtherapie der Psoriasis dar. Um die therapeutischen Leistungen besser abzubilden und auch um für die Patienten der Region neue moderne Behandlung optimal anzubieten, findet an der Universitäts-Hautklinik Kaunas ein monatliches Konsilium zwischen Dermatolo-
Tab.3 Die häufigsten Prozeduren an der Universitäts-Hautklinik Kaunas (im Jahr 2013).

\begin{tabular}{|l|l|}
\hline Prozeduren & Fallzahlen \\
\hline Biopsie ohne Inzision an Haut und Unterhaut & 2856 \\
\hline Ganzkörper-Lichttherapie $^{1}$ & 4293 \\
\hline Lokale Lichttherapie & 1654 \\
\hline Lokale Exzision von erkranktem Gewebe & 924 \\
\hline Digitale Dermatoskopie & 675 \\
\hline Epikutantestung von Allergenen & 510 \\
\hline Elektrokoagulation & 334 \\
\hline Kryotherapie & 372 \\
\hline Ultraschall der Haut und Unterhaut & 289 \\
\hline
\end{tabular}

1 UVB-311 nm-Lichttherapie betrug 78\%, Bade-PUVA 16\%, systemische PUVA 3\%, UVA 3\% der Gesamtzahl.

gen und Patienten mit mittelschweren und schweren Psoriasisformen statt. Im Jahr 2011 hat die staatliche Krankenkasse ein Budget von 1,1 Million Litas (etwa 314000 Euro) für die biologische Therapie an zwei Universitäts-Hautkliniken in Kaunas und Vilnius gewährt. Der humanisierte Anti-IL17-Antikörper und andere neue moderne Medikamente vor der Zulassung konnten durch laufende klinische Studien bei Psoriasis und atopischem Ekzem angeboten werden. 


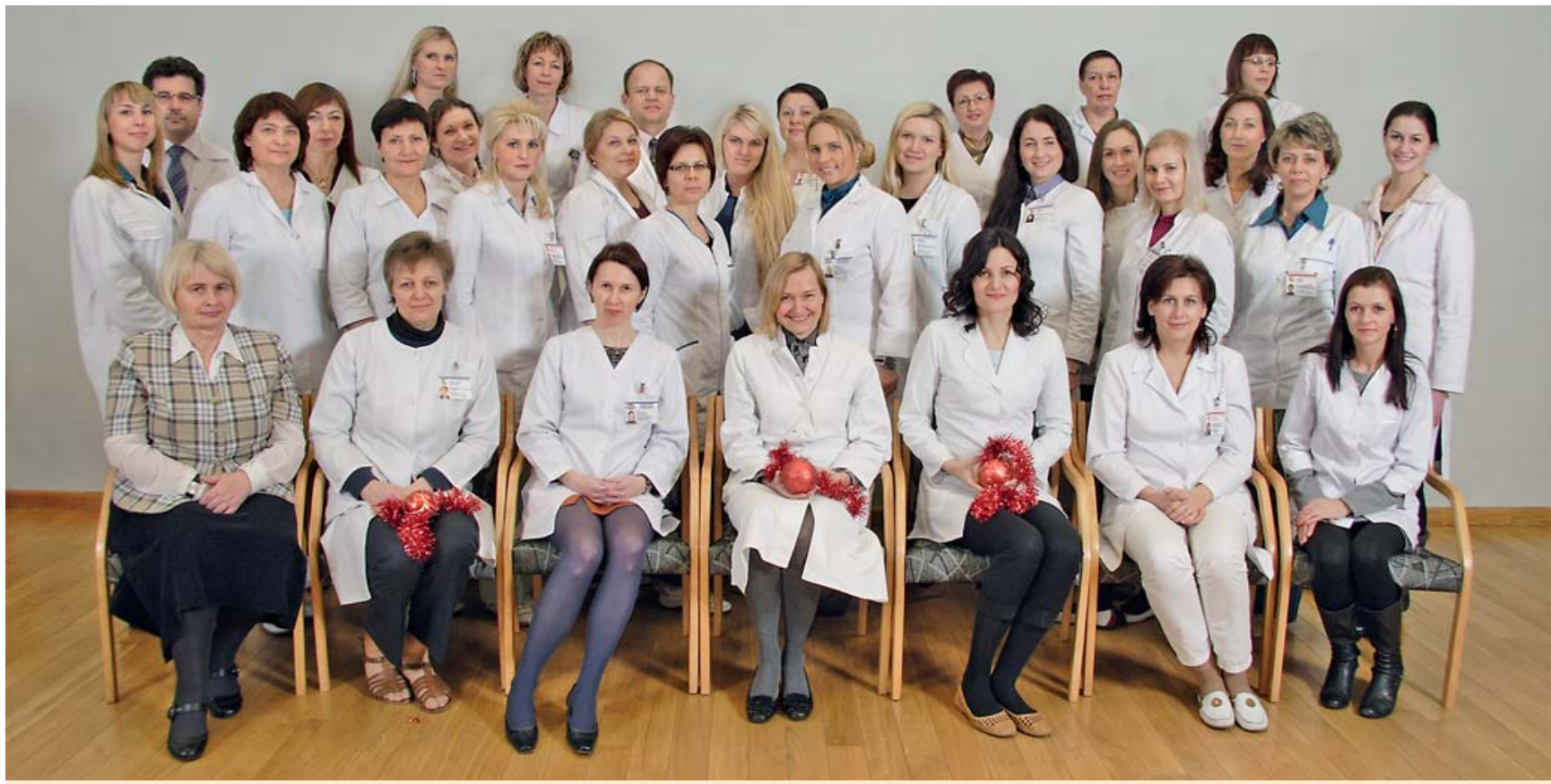

Abb. 1 Medizinisches Personal der Universitäts-Hautklinik Kaunas.

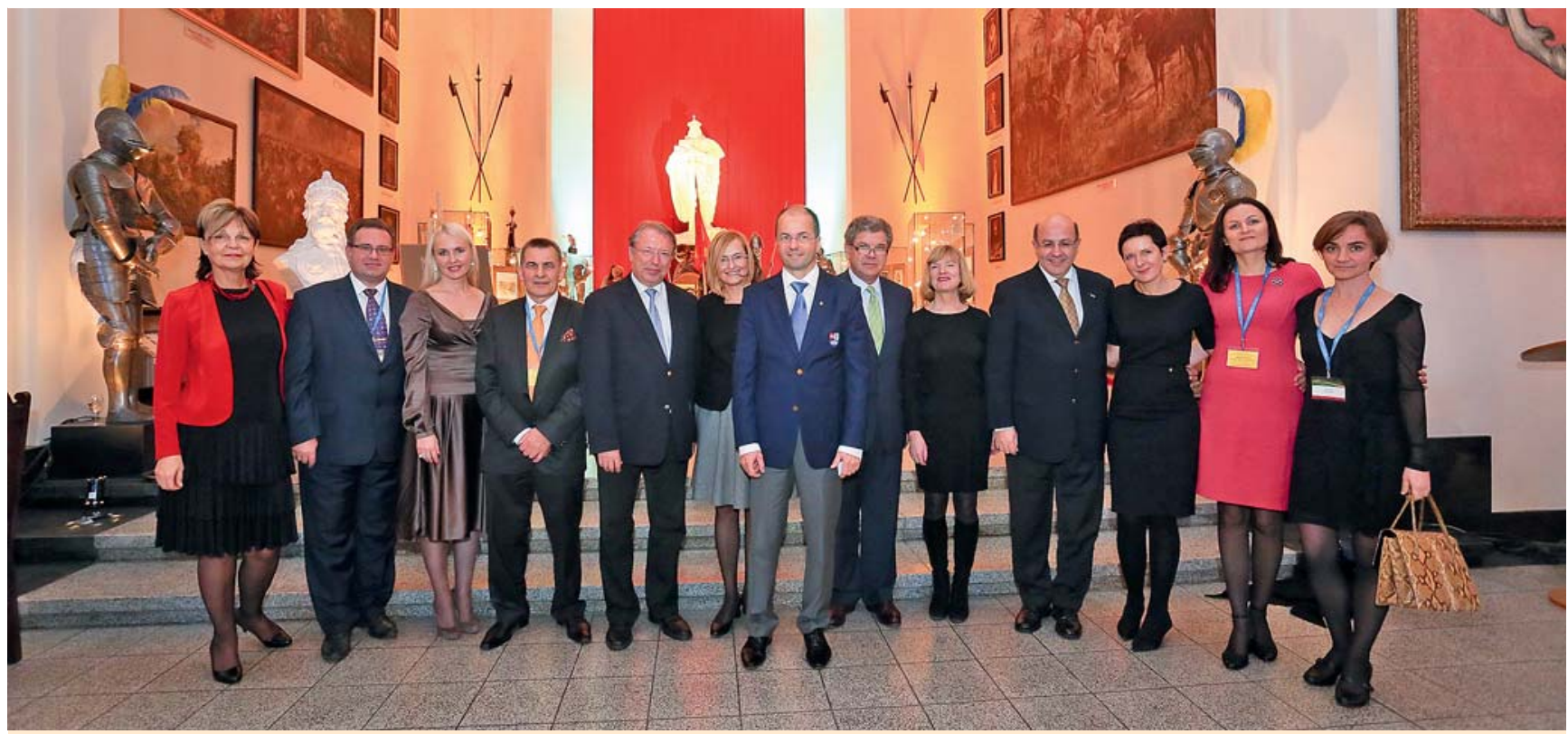

Abb.2 Die Dermatologen und der Rektor (Siebenter von links) der Litauischen Universität für Gesundheitswesen, Kaunas, bei der Eröffnung des Kongresses der Gesellschaft der Baltischen Dermatovenerologen im Kriegsmuseum von Kaunas. Erste von links: Airi Poder (Tartu), Jacek Szepietowski (Wrozlav), Matilda Bylaite (Vilnius), Andris Rubins (Riga), Claus Garbe (Tübingen), Skaidra Valiukeviciene (Kongresspräsidentin, Kaunas), Remigijus Zaliunas (Kaunas), Rudolf Stadler (Minden), Mona Stahle (Stockholm), Christos C. Zouboulis (Dessau), Ruta Ganceviciene (Vilnius), Aiste Beliauskiene (Kongresssekretärin), Cecilia Swedman (Malmö).

Die ärztliche Versorgung unserer Patienten wird derzeit von 26 Dermatologen gewährleistet (50\% davon in der Fachausbildung für Dermatologie und Venerologie). Die gesamte Zahl der Mitarbeiter der Universitäts-Hautklinik Kaunas umfasst etwa 50 Angestellte ( Abb.1). Die Klinik veranstaltet einmal pro Jahr einen Kongress der litauischen Dermatologen, angelehnt an den Welttag der Psoriasis, sowie dreimal pro Jahr eine dermatologische Weiterbildung der Dermatologen und Venerologen aus dem Einzugsgebiet um Kaunas. Im Jahr 2013 fand in Kaunas zum ersten Mal der 11. Kongress der Gesellschaft der Baltischen Dermatovenerologen (BADV) statt. An dem Kongress nahmen 300 Delegierte teil und 50 Vorträge rekrutierten sich aus insgesamt 35 Ländern ( Abb.2). Sechs deutschsprachige Professoren waren beteiligt: Claus Garbe (Tübingen), Wolf-Henning Boehncke (Frankfurt/Genf), Diamant Thaci (Lübeck), Tomas Ruzicka (München), Rudolf Stadler (Minden) und Christos Zouboulis (Dessau). 


\section{Forschungstätigkeiten}

$\nabla$

Die Forschungstätigkeiten der Universitäts-Hautklinik Kaunas umfassen epidemiologische und klinische Studien zu Hauttumoren, Akne, Kontaktekzem und Geschlechtskrankheiten. Seit 2009 nahmen die Dermatologen der Hautklinik Kaunas an zwei internationalen Projekten teil, Eurostar und Framework-7. Diese Projekte schließen die Etablierung der innovativen, bildgebenden Ultraschall-Technologien ein in einer Kombination mit der Thermografie, Dermatoskopie und Spektrofotometrie zur Verbesserung der Frühdiagnostik des diabetischen Fußes (www.skindetector.eu) und des malignen Melanoms der Haut. Seit 2012 beteiligt sich die Hautklinik am COST-Projekt „StanDerm“. Das Projekt fördert die Entwicklung und die Etablierung der Europäischen Standards und der Prävention von Berufsdermatosen. Seit 2013 wirken die Dermatologen an dem internationalen Projekt für die Verbesserung der Kontrolle der Geschlechtskrankheiten in den baltischen Ländern und Ost-Europa mit. Bei der Durchführung der Forschungsprojekte arbeitet die Hautklinik eng zusammen mit dem Ultraschall-Institut der Technischen Universität Kaunas, dem ESSCA (European Surveillance System on Contact Allergy), dem CVDerm Zentrum (Hamburg), der Otto-von-Guericke-Universität Magdeburg und der Universität Halle (Akne-Studien und Naevi-Studien „PANS“) sowie mit SIDA (Swedish International Development Cooperation Agency).

\section{Fortbildung der Studenten \\ $\nabla$}

Die Litauische Universität für Gesundheitswesen (bis 2010 Medizinische Universität Kaunas) ist die größte Hochschule für das Biomedizinstudium in Litauen und entsprechend ist das Universitätsklinikum das größte Krankenhaus des Landes. Im Jahr 2013 wurden die Dermatologie und Venerologie an der UniversitätsHautklinik Kaunas 350 Medizin-Studenten des 4. Studienjahres (60 Kontaktstunden), 142 Odontologen (30 Kontaktstunden) des 5. Studienjahres, 56 Nurses des 1. - 3. Studienjahres (40 Kontaktstunden) und 21 Public Health-Studenten des 2. Studienjahres (10 Kontaktstunden) gelehrt. Dies gründet sich auf der seit 1999 zunehmenden Aktivität des wissenschaftlichen Zirkels der Medizin-Studenten zugunsten der Dermatologie und Venerologie. 2013 lag die Zahl der jungen Wissenschaftler in der Dermatologie bei 31. Im Jahr 2002 wurde die Facharztausbildung in der Dermatologie und Venerologie von zwei auf vier Jahre verlängert. Jedes Jahr bietet die Universitäts-Hautklinik Kaunas 3 neue Facharztausbildungsplätze für Dermatovenerologie an.

\section{Zusammenarbeit mit deutschsprachigen Dermatologen \\ $\nabla$}

Die Partnerschaften mit deutschsprachigen Ländern wurden seit 1998 vielseitig erneuert. Die Universitäts-Hautklinik Magdeburg unter ihrem Direktor Harald Gollnick entwickelte sich zu einem Zentrum der wissenschaftlichen Beziehungen. Zwei Promotionen in den Bereichen der Dermatoonkologie (2002) und Dermatohistopathologie (2005) an der dermatologischen Universitätsklinik Kaunas unter H. Gollnicks Leitung waren der sichtbare Be- ginn dieser Gemeinsamkeit. Viele Assistenten wurden in verschiedenen dermatologischen Gebieten an der Universität Magdeburg weitergebildet ( Tab.1). Das Prinzip einer interdisziplinären Dermatoonkologie wurde an der Universität Kaunas eingeführt. Mit Unterstützung von Jens Ulrich, Magdeburg/Quedlinburg, wurde seit 2003 die Sentinel-Lymphknoten-Biopsie für das Staging von Hautmelanomen aufgebaut. Nach der Weiterbildung von zwei Pathologen an der Universität Magdeburg wurde am Universitätsklinikum Kaunas die immunohistochemische Diagnostik für Hautlymphome vervollkommnet.

In den zehn Jahren der Partnerschaften bekam die dermatologische Universitäts-Hautklinik Kaunas mehrmals die Förderung der Deutschen Dermatologischen Gesellschaft (DDG), der Berliner Stiftung für Dermatologie, des European Surveilance System of Contact Allergies (ESSCA) sowie von den universitären dermatologischen Kliniken Magdeburg (Direktor: Harald Gollnick), Basel (Vorstand: Theodor Rufli) und München-Schwabing (Direktor: Wolfgang Stolz). Eine Vielzahl von Büchern wurde der Klinikbibliothek geschenkt (hrsg. von B. Bonnekoh, H. Gollnick, Constantin E. Orfanos, T. Rufli, sowie als eine Stiftung von O. Braun-Falco/München, G. Mahrle und E. Friedemann/Werdohl). Die Dermatologen der Universitätsklinik Kaunas sind für die ständige und vielseitige Unterstützung des Faches den deutschsprachigen Kollegen sehr dankbar.

\section{Interessenkonflikt}

$\nabla$

Die Autorin gibt an, dass kein Interessenkonflikt besteht.

\section{Abstract}

\section{A Clinic Presents itself - The University Clinic for Dermatology Kaunas, Litauen $\nabla$}

In 1923, the first Department of Skin and Venereal Diseases was created at Vytautas Magnus University in Kaunas. In 2001, the Department of Skin and Venereal Diseases was removed to the University Hospital Kaunas Clinics. Since that moment the influence of the cooperation with German-speaking dermatologists and the multidisciplinary approach in the hospital led the Department of Skin and Venereal Diseases to progress. The new opportunities opened the field for Dermatooncology, Dermatologic Surgery and Photochemotherapy. The continually increasing number of dermatological patients, students and residents shows that the Department of Skin and Venereal Diseases in Kaunas has become an important centre of dermatological and venerological care.

\section{Literatur}

1 Valiukeviciene S, Barstiene V, Lignugariene A. Weg der Dermatologie in Litauen. Akt Dermatol 2013; 38: 130-133

2 Valiukeviciene S, Lignugariene A. Dermatology in Lithuania. In: Scholz A, Holubar K, Burg G et al., ed. History of German Language Dermatology. Darmstadt: Wiley-Blackwell; 2009: 627-637 